\title{
Habitats pérennes ou précaires au Néolithique
}

Perennial or precarious settlement in the Neolithic

\section{Alain Beeching}

\section{(2) OpenEdition}

\section{Journals}

Édition électronique

URL : https://journals.openedition.org/tc/5333

DOI : $10.4000 /$ tc. 5333

ISBN : 1952-420X

ISSN : 1952-420X

Éditeur

Éditions de l'EHESS

Édition imprimée

Date de publication : 5 septembre 2011

Pagination : 48-61

ISBN : 978-2-7351-1410-8

ISSN : 0248-6016

\section{Référence électronique}

Alain Beeching, « Habitats pérennes ou précaires au Néolithique », Techniques \& Culture [En ligne], 56 |

2011, mis en ligne le 30 novembre 2011, consulté le 29 septembre 2022. URL : http://

journals.openedition.org/tc/5333; DOI : https://doi.org/10.4000/tc.5333 


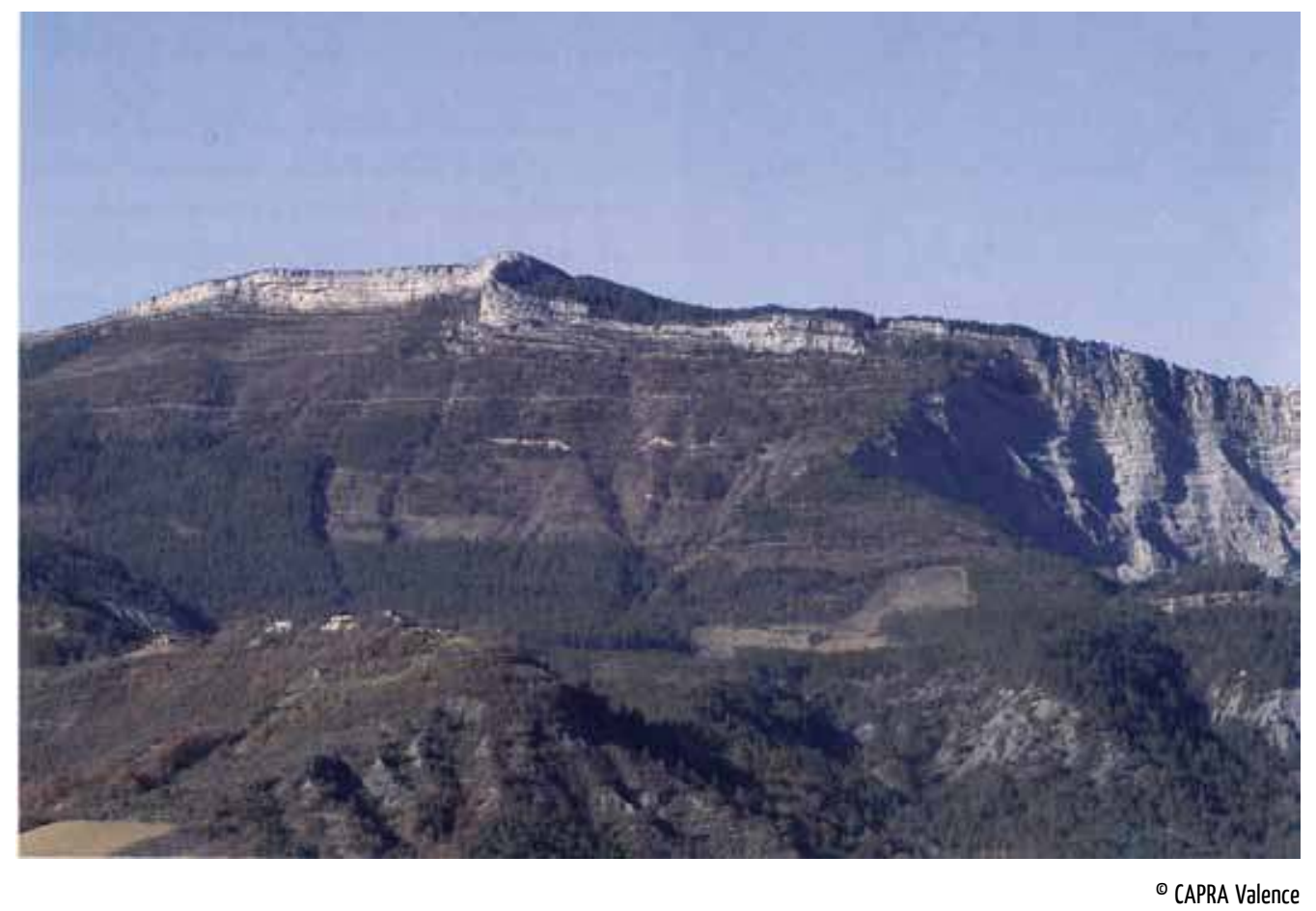




\section{HABITATS PÉRENNES OU PRÉCAIRES AU NÉOLITHIQUE}

Faut-il rappeler que l'Archéologie, comme ensemble de méthodes et techniques d'investigation, seule pourvoyeuse de données pour l'étude de la Préhistoire, ne peut s'appuyer que sur des faits matériels (objets mobiliers, traces, déplacement ou agencement de la matière) et les analyses plus ou moins sophistiquées qui en découlent? On cherche à étendre ce champ du monde physique, d'abord appréhendé, par des comparaisons, recoupements, raisonnements, productions d'hypothèses interprétatives, par le recours à la logique, au comparatisme avec des situations analogues dans d'autres champs historiques ou culturels mieux connus, à l'expérimentation, etc. Mais les matériaux de base de l'analyse restent du ressort du monde physique. Certains domaines d'investigation en sont favorisés : étude de l'objet, des rejets culinaires, des comportements funéraires, de l'habitat ... Il pourrait sembler que ce dernier soit donc aisément abordé, puisque résultant par excellence de la transformation et de l'aménagement de la matière. Ce n'est pourtant pas le cas. Les exemples de structures bâties, clairement lisibles en plan grâce à des traces au sol (bâtiments sur poteaux, soubassements empierrés), ne sont pas les plus fréquents. Derrières quelques représentations emblématiques, constamment reprises dans les écrits de vulgarisation, se cachent une majorité de vestiges tronqués, de traces peu lisibles et difficilement interprétables. La connaissance que l'on peut en tirer de l'organisation de vie des sociétés anciennes en souffre inévitablement ; si on peine le plus souvent à dégager pour chacune d'elles un archétype de maison, on est encore plus éloigné d'en saisir les variations. Il y a heureusement des situations où la convergence d'informations, mêmes incomplètes, permet d'appréhender ces variantes et de commencer à réfléchir sur ce qu'elles nous disent de la complexité interne des sociétés elles-mêmes. 
Nous proposons d'examiner ici une série de faits disjoints dans le temps et l'espace mais qui concernent tous le stade historico-culturel dit Néolithique pendant lequel les communautés humaines de certaines régions du monde ont accédé pour la première fois à l'économie de production alimentaire et/ou à des évolutions techniques et sociales décisives (Demoule 2009). Parmi ces dernières, le regroupement de populations en agglomérations plus stables (paradigme assez flou de la sédentarisation) est souvent avancé. Ce fait, maintes fois mis en évidence par l'archéologie, mérite souvent d'être nuancé et complété, pour en expliquer le mode de fonctionnement, par la prise en compte de sites réputés secondaires, participant d'un réseau de circulations et d'activités complémentaires.

\section{Vestiges, techniques de construction, statut de l'habitat}

On n'insistera pas longuement sur le point de départ de la réflexion. On voit bien les pièges du raisonnement selon lequel des vestiges ténus signifieraient un habitat précaire à préciser (provisoire, annexe, mobile...). Avec précautions, l'archéologie est cependant contrainte de hiérarchiser les faits rencontrés en prenant en compte : le groupement, la taille, les techniques, les matériaux, et quand elle le peut (par exemple dans le cas de l'habitat en milieu humide où on peut s'appuyer sur les mesures fines de la dendrochronologie) la durée... des bâtiments mis en évidence. Aucun fait ne peut être en soi exclusif ou décisif, mais le sens de l'examen est souvent d'évaluer l'investissement requis en moyens et en temps, pour juger de l'importance et du statut d'un lieu habité. Même connue et maîtrisée, la tendance mécaniste de concevoir une corrélation et un accroissement conjoint de la « lourdeur » d'un bâtiment et de son « importance » en termes de durée et de fonction, est certaine. L'archéologie du Néolithique livre de nombreux cas d'une extrême variabilité. S'il n'est pas possible de comparer de ce point de vue des entités culturelles différentes, la question se pose sur des bases très directement quantitatives au sein de chacune d'elles, quand c'est possible. Une des difficultés majeures est d'associer une adaptation de l'habitat à une ou des activités précises; et quand on discerne des activités spécifiques dans des sites différents, une autre est de savoir si il y a un lien entre eux ; et de quelle nature. Sans lien direct entre les deux, sinon dans le raisonnement, nous examinerons deux séries de faits permettant d'aborder ces questions.

\section{L'exemple de la néolithisation au Proche-Orient}

Entre le $\mathrm{x}^{\mathrm{e}}$ et le viI ${ }^{\mathrm{e}}$ millénaires avant notre ère, se forme et se développe, au Proche-Orient, un des quelques foyers autonomes de passage au Néolithique dans le monde, le mieux connu par l'abondance des travaux et publications qu'il a suscité (Aurenche et Kozlowski 1999). Les modalités et les étapes de la « néolithisation » de ces régions (essentiellement Sud de la Turquie, Syrie, Iraq, côte levantine, vallée du Jourdain) sont assez bien connues dans les grandes lignes. Dès le stade mésolithique des derniers chasseurs-cueilleurs du Natoufien, aux xII ${ }^{e}$ et XI ${ }^{e}$ millénaires (Bar Yosef \& Valla 1991) on assiste, dans cette région, 
à un phénomène de sédentarisation relative, marqué archéologiquement par des vestiges de premiers hameaux, comportant une demi-douzaine de maisons circulaires ou semicirculaires de 5 à $12 \mathrm{~m}$ de diamètre, dont la base des parois, partiellement enterrées, est renforcée par une couronne de blocs de pierres, parfois plusieurs assises superposées. Dans certains cas, des sols damés, des calages de poteaux soignés, la présence de matériel de meunerie lourd, de silos et de sépultures démontrent, pour les spécialistes de cette période, l'enracinement partiel de ces premières agglomérations. C'est particulièrement clair dans certaines zones géographiques, plus propices par leurs ressources naturelles, à ce début de fixation sédentaire. Mais il existe des sites de même époque où les critères d'ancrage sont moins probants, peut-être en liaison avec des ressources naturelles moins abondantes, et on évoque une variabilité circonstancielle ou saisonnière de cette fixation au sol (Aurenche \& Kozlowski ibid.).

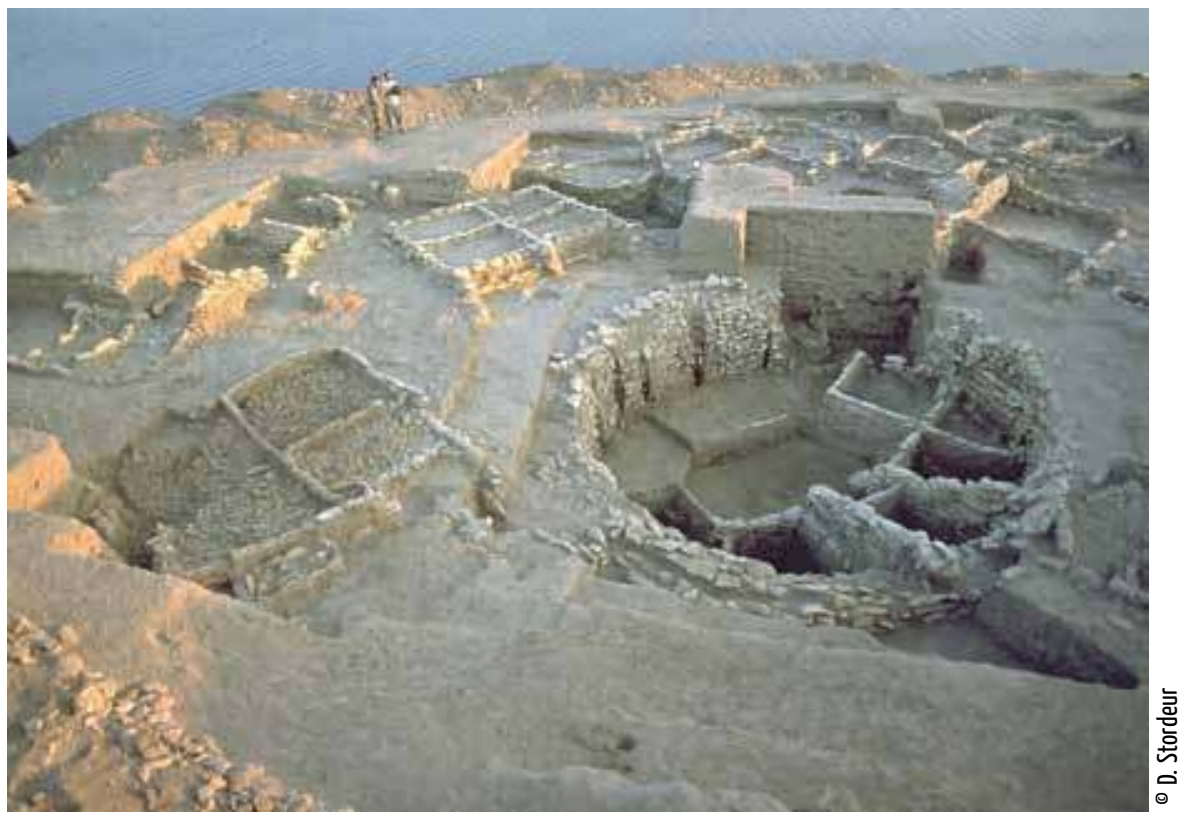

Avec le processus de néolithisation, en prolongement de la cueillette et de la chasse sélective, on voit l'apparition et le développement progressif de l'agriculture et de l'élevage. Toujours dans ces zones favorisées que représentent les vallées des grands cours d'eau et les piémonts des massifs montagneux, la multiplication des tells, ces superpositions d'habitats dues à une pérennité ou à un retour répété au même endroit des groupements humains, montrent une extension considérable de la taille des agglomérations au cours des étapes dites PPNA (Pre-Pottery Neolithic A : 10000 - 8800 environ av. notre ère) et PPNB (Pre-Pottery Neolithic B : 8800 - 7000); cette dernière voyant le succès avéré de ces techniques de production alimentaire. À Jerf el Ahmar ${ }^{2}$ (Syrie), le site (Figure 1)actuellement le mieux connu pour le PPNA (Stordeur 1999, 2000), neuf niveaux permettent d'observer une évolution progressive de l'architecture des maisons à murs

\section{Jerf el Ahmar (Syrie)}

Vue du niveau II (PPNA, Néolithique précéramique, 9500 à 8500 av. notre ère) du tell Ouest. Autour d'un bâtiment rond enterré, considéré comme communautaire, se répartissent les maisons de plan quadrangulaire à angles plus ou moins droits ou arrondis, à soubassement de plusieurs assises de pierres, subdivisées en plusieurs cellules. C'est un exemple des occupations répétées et durables qui se succèdent cycliquement au même endroit pendant des siècles ou millénaires dans plusieurs tells des bords de l'Euphrate.

(Fouilles D. Stordeur, Maison de I'Orient et de la Méditerranée, Lyon). (Fig. 1) 


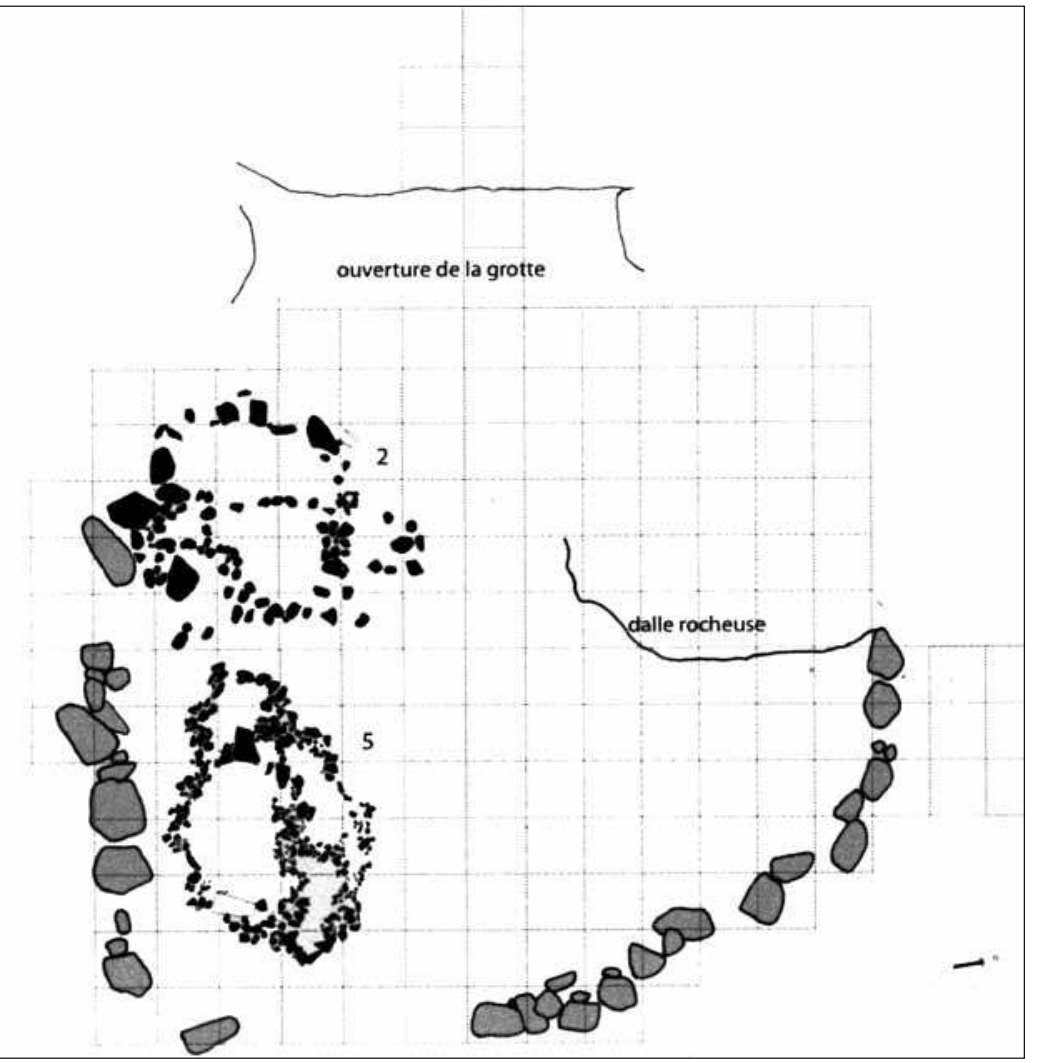

Abri de Wadi Tumbaq 1 (montagnes du Ba'las, Syrie)

Plan des vestiges de deux constructions. La structure 5 , pluricellulaire est attribuée au PPNA ( $X$ e et début du IXe millénaires avant notre ère), édifiée devant l'abri en zone montagneuse aride (Quadrillage métrique. Relevé F. Abbes, Maison de I'Orient et de la Méditerranée, Lyon).

(Fig. 2) de pierres d'un plan circulaire unicellulaire ou à divisions fonctionnelles à un plan quadrangulaire à angles arrondis puis anguleux et chaînés, subdivisé en trois ou quatre pièces. Le sens de cette évolution est confirmé par plusieurs sites du PPNB ancien et moyen attestant un accroissement de la superficie de villages sédentaires (plusieurs hectares), un investissement de plus en plus important dans les travaux d'aménagement communautaires et dans les techniques de construction en terre, pierres et terre, brique, la probabilité d'espaces collectifs... Avec des variantes, s'impose un modèle d'habitat et une forme de normalisation de son organisation interne, majoritairement tripartite, sur la base des fonctions qui lui sont assignées : à l'avant, aire d'activités et de transformation; au centre, la grande pièce à vivre avec four et foyers mais aussi sépultures enterrées ; à l'arrière, zone de stockage (Molist Montana 2001).

Mais, à côté de cet ancrage sédentaire et de cette concentration humaine croissants, rendus possibles par l'exploitation alimentaire prédatrice raisonnée puis productrice mais toujours dans des contextes écologiques favorables, on note la présence d'habitats plus «précaires », selon la définition proposée plus haut, dans des zones arides ou semi-arides du Proche-Orient. C'est le cas dans le désert syrien de la Palmyrène et notamment dans la région montagneuse du Ba'las où Frédéric Abbes mène depuis plusieurs années un important travail ${ }^{1}$. Sur les hauteurs du vallon du Wadi Tumbaq, s'ouvrent de nombreuses cavités et abris occupés à toutes les périodes. Pour les périodes considérées ici, notamment, l'abri de Wadi Tumbaq 1 (Figure 2) a livré une succession d'occupations s'échelonnant entre la période dite Khiamienne (pré-néolithique) et la fin du PPNB. Les traces d'habitat montrent une évolution morphologique conforme à l'évolution architecturale définie pour les sites sédentaires, nécessitant parfois un investissement collectif important (construction d'une terrasse artificielle régulièrement entretenue) mais présentant le plus souvent des vestiges plus fugaces.

Linterprétation archéologique de vestiges matériels, mobiliers et immobiliers, est délicate. Pour le Khiamien, la présence d'objets de divers statuts fonctionnels (rejets osseux, chaîne du débitage du silex, récipients en pierre, parures...) montre qu'il s'agit d'habitats « complets », mais peut-être temporaires... la durée restant difficile à estimer et la comparaison malaisée à établir faute de documentation conséquente. Pour le PPNA, on retrouve, avec une mise en œuvre moins lourde et sous une forme simplifiée cependant très claire, la transposition du plan tripartite, voire quadripartite, et de l'organisation cloisonnée, de l'espace des villages sédentaires. La quantité et la nature des vestiges conduisent l'interprétation vers une succession de campements saisonniers essentiellement tournés vers la chasse. En zones arides, la mobilité n'est donc pas abandonnée à ce moment décisif de sédentarisation croissante en zones propices, mais on voit bien, dans la morphologie de l'habitat, un lien entre les deux. 
On peut alors signaler que c'est sans doute vers la fin de ce PPNB que s'instaure un mode de vie appelé à une longue postérité dans ces régions : le nomadisme pastoral. De nombreux travaux et réflexions ont été consacrés à ce phénomène apparaissant sur les marges désertiques des zones les plus favorables (Cauvin 1990 ; Francfort 1990 ; Alarashi 2005). On ne rentrera pas ici dans le détail des situations connues par l'ethnologie et extrapolées par hypothèse dans les scénarios explicatifs de l'archéologie. Les classements et débats portent sur plusieurs critères : le statut et la durée des haltes observées, dans des systèmes de circulations itinérantes ou rayonnantes, en autarcie ou à liaison avec un point central fixe, selon un pastoralisme exclusif ou en combinaison avec une agriculture d'appoint sommaire, etc. Dans les cas concernés du Néolithique proche oriental, la discussion tente de démêler d'après la nature et l'importance des vestiges : la taille des groupes humains, leur durée de résidence, les types de structures habitées et utilisées, les activités pratiquées. Quantitativement, la plupart des données vont dans le sens de groupes restreints et de haltes brèves. Qualitativement, on est loin de la diversité de mise dans des activités domestiques « complètes », ce qui semble indiquer une activité spécialisée en liaison avec un pôle central situé ailleurs. Concernant les activités pratiquées; sur plusieurs sites (par exemple El Khabra, cuvette d'El Kowm, Syrie), la présence de pointes de flèches laisse entrevoir une activité de chasse. Sur d'autres (par exemple à Qdeir 1, même localisation), on retient une très nette sous représentation des artefacts traduisant une activité agricole (une meule, rares éléments de faucilles), mais par contre la présence de restes fauniques attestant la consommation et l'élevage des caprinés (moutons et chèvres) et du bøuf, avec un abattage concentré sur les saisons du printemps et de l'automne, ainsi que la chasse de la gazelle principalement (Gourrichon 2004). Les structures associées aux sites sensibles sont rares. On retrouve les plans cloisonnés irréguliers évoqués plus haut (sites de Jilat 13, bassin d'Azraq en Jordanie, un peu plus récent) mais on connaît dans les zones désertiques d'autres types de structures mal datées qui pourraient coïncider avec cette période : des enclos de diverses tailles et formes ayant pu servir à la gestion des troupeaux.

On voit donc, au bilan de cet exemple, qu'il existe dès les premiers millénaires de l'instauration de l'économie de production au Proche-Orient, une catégorie d'implantations qui s'écarte très sensiblement de la norme en cours de fixation dans les grands villages tout en en gardant la trace et probablement parfois un lien de complémentarité économique. Cet habitat provisoire et les structures qui lui sont liées sont voués à répondre à un besoin fonctionnel spécifique, même si celui-ci reste variable et parfois imprécis.

\section{Grottes-bergeries et sites centraux dans le Néolithique rhodanien}

Cet exemple nous est plus proche de quelques millénaires mais reste archéologiquement rattaché au stade Néolithique. Les innovations vues au Proche-Orient ont gagné peu à peu vers l'Ouest, par la Méditerranée et par les plaines de l'Europe centrale pour parvenir sur le sol de la France actuelle vers le milieu du VIe millénaire avant notre ère. Dans le Sud, le premier impact est encore en phase adaptative avec des survivances du stade Mésolithique mêlées aux nouveaux comportements économiques. Les sites sont nombreux mais les structures d'habitat sont rares. Cet état de fait perdure et s'intensifie 


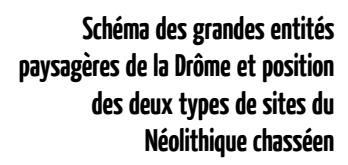

Les grottes-bergeries et les très grands sites de terrasses alluviales : leur complémentarité, depuis longtemps envisagée, a été prouvée par celle des courbes d'âges d'abattage des caprinés (D.A.O. A. Beeching).

(Fig. 3)

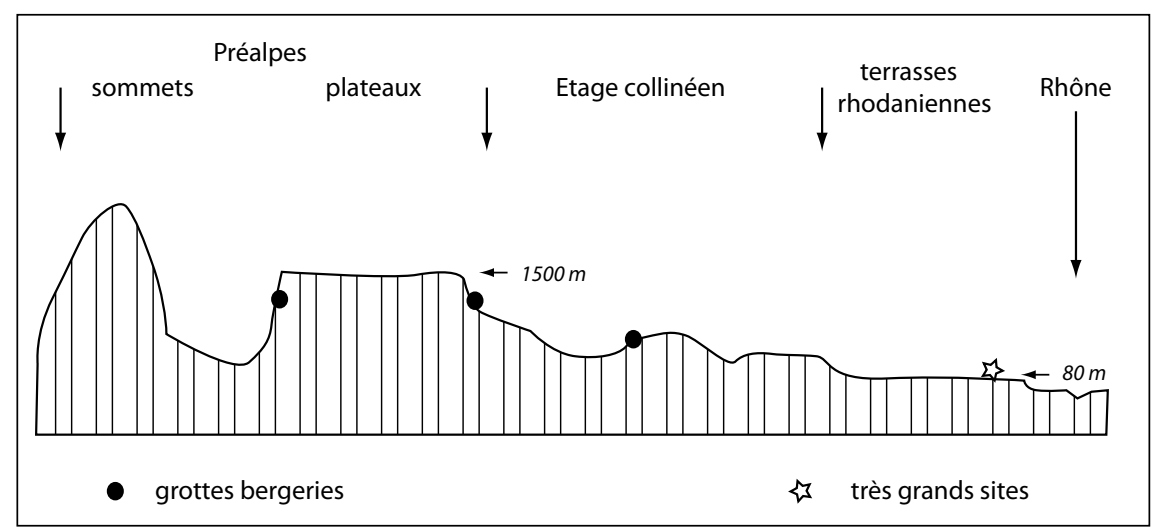

lors de l'étape suivante. À partir de 4250 av. notre ère environ et pendant près de sept siècles, se développe le complexe culturel dit «Chasséen » (Beeching \& al. 1991). Les faits et comportements reconnus par l'archéologie de cette période restituent le tableau d'une société complexe, expansive, marquant des avancées techniques dans tous les domaines, notamment dans la production des objets manufacturés : céramique, silex, os, etc. Lors de ses phases les plus récentes (à partir de 4000 environ), ce Chasséen méridional présente dans le bassin du Rhône moyen, une série de traits qui concernent le sujet débattu et que nous allons détailler un peu (Beeching \& Brochier 1990 ; Beeching \& al. 2000 ; Beeching 2003).

Dans le seul département de la Drôme, entre Rhône et Préalpes calcaires, le Chasséen est présent sur 150 sites environ, dans l'état actuel des recherches. Ceux-ci se répartissent dans la quasi-totalité des entités géographiques et des positions topographiques, depuis les basses terrasses du fleuve et de ses affluents jusqu'à la moyenne montagne, au-dessus de 1500 mètres d'altitude, en plein air et en grottes (Figure 3). L'ordonnancement par classes de superficie et par positions topographiques montre une très grande diversité d'implantations, forcément liée à des contraintes, choix et activités diverses. L'étage collinéen, intermédiaire entre plaine et montagne est paradoxalement le plus fourni mais le plus mal connu, les prétextes à fouilles y étant plus rares : ni grands travaux préventifs de la première, ni position très typée attractive et circonscrite (grottes, positions de cols) de l'autre. Deux types de sites exceptionnels sortent du lot : les très grands sites de terrasses et les grottes-bergeries.

\section{Les très grands sites des terrasses rhodaniennes}

Ces grands sites sont peu nombreux, trois ou quatre pour la Drôme, et de superficie considérable, souvent difficile à arrêter précisément. Deux de ces sites ont été sondés et longuement fouillés lors de multiples opérations et permettent d'en évaluer l'importance : à Saint-Paul-Trois-Châteaux (travaux entre 1984 et 2010, d'une dizaine d'hectares au moins) et sur le site du Gournier à cheval sur les communes de Montélimar et Châteauneuf-du-Rhône (travaux entre 1976 et 2010, de 250 hectares au moins) (Beeching \& al. 2010).

Plusieurs critères les caractérisent. Il s'agit de sites où les sols archéologiques sont extrêmement mal conservés, voire souvent absents, à la suite d'activités érosives multiples 
et complexes à diverses époques. En dehors de ces lambeaux ou d'accumulations de sédiments dans des chenaux fossiles, l'essentiel de la documentation consiste en des organisations structurées par l'homme, dispersées sur de grandes surfaces, parfois groupées :

- empierrements circulaires de $1 \mathrm{~m}$ environ de diamètre, résultant d'amas volontaires de blocs et galets, parfois liés à des foyers à pierres chauffantes.

- fosses circulaires de toutes morphologies, affectant parfois la forme de silos (grand volume et ouverture étroite) ; parfois fosses moins profondes et de contours plus irréguliers

- inhumations humaines en fosses étroites ou en silos, le plus souvent à corps unique mais quelques fois multiples, à plus de 3 et jusqu'à 9 corps, en des rituels d'organisation complexes. Quand l'observation le permet, on perçoit parfois des organisations spatiales associant sépultures, empierrements et fosses. On émet l'hypothèse qu'une certaine scénarisation règle cette disposition, dans le cadre de cérémonies à caractère social pendant et après les enterrements (Beeching 2003).

L'étude des restes osseux animaux, abondants dans ces fosses, montre une découpe de type bouchère et une consommation culinaire des caprinés (moutons et chèvres) et des bovinés principalement. Mais plusieurs anomalies sont parfois perceptibles par rapport à l'hypothèse la plus simple d'une consommation de type domestique et villageois : animaux consommés inhabituels (chiens à Saint-Paul-Trois-Châteaux), concentration des restes dans certaines fosses, parfois choix des parties anatomiques, absence de certaines classes d'âge (individus très jeune et bêtes de réforme) laissant apparaître une courbe d'abattage tronquée par rapport à celle d'une population viable. Même si certains secteurs du Gournier peuvent paraître complémentaires, il manque manifestement des restes osseux (Bréhard 2007 ; Bréhard \& al. 2010).

Un autre trait caractérise ces grands sites, l'extrême rareté des traces d'habitat. À Saint-Paul-Trois-Châteaux, aucun « trou de poteau » (empreinte en creux caractéristique), qui signerait un bâtiment sur poteaux bloqués, c'est-à-dire fichés en terre, aucune trace en négatif, aucun effet de paroi (lignes de pierres, limites de répartition d'objets, ...) qui indiquerait, faute de plan clair d'un bâti, l'emplacement possible de celui-ci ... $\mathrm{Au}$ Gournier, deux emplacements de bâtiments sont probables : l'un, signalé par une série de trous de poteaux inorganisée, n'offre pas de plan convaincant mais pourrait occuper plusieurs dizaines de mètres carrés (lieu-dit Fortuneau 2006, zone B, direction et renseignements F. Cordier ${ }^{3}$ ) ; l'autre, matérialisé par une chape d'argile rapportée et des traces de ruissellement formant effet de paroi, est de plan circulaire, de cinq mètres de diamètre et d'une vingtaine de mètres carrés de superficie. Au bilan, c'est quantitativement très peu
Une vue du plateau du Vercors

Exemple des étendues herbeuses de moyenne montagne près desquelles sont souvent situées les grottes-bergeries néolithiques. (Fig. 4) 
pour des sites de cette importance, puisque ces quelques cas prouvent qu'il ne s'agit pas d'une absence due à des problèmes de conservation. Qualitativement, il s'agit de vestiges extrêmement ténus. Les superficies, le faible diamètre des poteaux dans un cas, leur absence dans l'autre, laissent supposer des structures bâties de faible importance qui, si l'on se réfère aux modes de raisonnements esquissés plus haut, sembleraient plutôt indiquer un habitat fugace ou temporaire qu'une implantation pérenne lourde. Mais le problème, dans le cas du Chasséen, est que l'on n'a pas ou peu de références de ces structures pérennes pour évaluer le « poids » de celles-ci. C'est clairement un exemple archéologique d'indécision faute de données. Pour tout le Chasséen méridional (près du quart de la France en superficie), pour lequel plus d'un millier de sites sont probables si on extrapole l'exemple drômois, moins d'une dizaine ont livré des traces de bâtiments, d'après la littérature spécialisée ; mais, si on en retranche tous les cas à problèmes (mal datés ou incomplètement décrits), il n'en reste plus que quatre sur lesquels s'appuyer (Beeching 1999 ; Saintot \&t al. 2009). On peut cependant envisager un archétype de bâtiment dont les constantes et variantes seraient les suivantes : plan quadrangulaire ou quadrangulaire à abside de 50 à $150 \mathrm{~m}^{2}$ environ, sur poteaux porteurs bloqués; ce qui est sensiblement différent des maigres vestiges des grands sites de terrasses.

Le statut de ces grands sites et, plus largement, le mode de vie et de résidence des populations du Chasséen méridional mériteraient de plus longs développements, notamment chronologiques, qui sortiraient du cadre de ce travail. Pour la suite du discours, on retiendra seulement que ces grands sites drômois ne se présentent pas, archéologiquement, comme de grands villages concentrant la population, les activités domestiques, économiques, sociales... La présence humaine y paraît plus espacée et intermittente que groupée et pérenne.

\section{Les grottes-bergeries}

Elles ont été mises en évidence il y a une petite trentaine d'années sur la base de plusieurs critères osseux, sédimentaires et archéologiques. Il s'agit le plus souvent, dans les Préalpes drômoises, de petites cavités à proximité de vastes plateaux ou étendues herbeuses (Figure 4) potentielles peu visibles (Figure 5), situées entre $1000 \mathrm{~m}$ et $1500 \mathrm{~m}$ d'altitude. Une dizaine de grottes-bergeries ont été identifiées; quelques unes fouillées et étudiées (Brochier \& Beeching 1999 et 2008). L'espace disponible, volontiers étroit et bas de voûte, n'est pas favorable à la vie permanente d'un groupe. Les fouilles et les études ont montré que le sédiment constitutif des phases d'occupations chasséennes résultait de la décomposition d'amas importants de fumiers animaux, mélanges de litières et de déjections, les fèces de caprinés étant parfois conservées et abondantes. Ces amas sédimentaires dont il reste surtout la partie minérale sont finement lités en témoignage de multiples phases de passage et d'absence des troupeaux que l'on peut, sans pouvoir le chiffrer précisément, estimer à plusieurs dizaines voire centaines de bêtes. Le cycle de fréquentation est saisonnier. Les vestiges mobiliers recueillis sont extrêmement peu nombreux à l'emplacement des parcages : quelques artefacts suffisant pour une attribution chrono-culturelle, des restes osseux notamment des dents de chute de très jeunes caprinés (Helmer \& al. 2005). Les traces et vestiges immobiliers témoignant de l'organisation de l'espace par l'homme sont également très minces. Il s'agit presque exclusivement de petits foyers à plat ou en cuvettes, n'excédant pas $0,60 \mathrm{~m}$ à $0,80 \mathrm{~m}$ de diamètre, recourant parfois à quelques pierres de chauffe ou de calage ; sûrement pas plus d'un ou deux par sol d'occupation. Il ne 
peut s'agir là de l'aménagement nécessaire à la vie domestique d'un groupe autarcique. Seules quelques personnes devaient accompagner le troupeau : les bergers par exemple. Signalons pourtant, pour être complet, le cas particulier d'une de ces grottes-bergeries (le Trou-Arnaud à Saint-Nazaire-le-Désert, Drôme) où, au contraire, s'accumulaient de grandes quantités de céramiques, et pour lequel on envisage une fonction de grotte-refuge.

Ces deux types de sites n'avaient rien en commun, sinon d'appartenir globalement au même courant culturel. La tentation de les relier dans un schéma de complémentarité se heurtait au risque élevé d'un décalage chronologique au sein d'un même complexe culturel. Les preuves indispensables à leur rapprochement

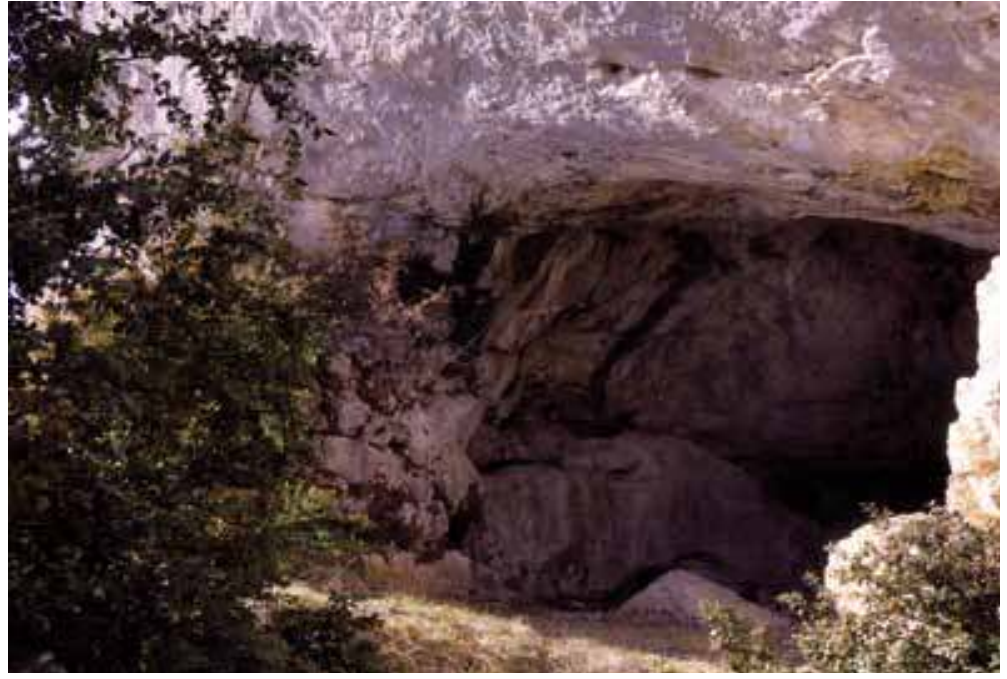

๑ J.-L. Brochier - CAPRA Valence sont venues des études archéozoologiques, des grottes-bergeries d'abord (Helmer $\&$ al.,

ibid.), des grands sites de terrasses ensuite (Bréhard 2007). La démonstration de leur complémentarité fonctionnelle (Bréhard \& al. 2010) repose sur la comparaison des courbes d'âges d'abattage des animaux sur ces sites, attestées par les restes osseux trouvés, principalement les dents. Pour les caprinés (moutons et chèvres), les cavités correspondent à un système de gestion du troupeau tourné vers la production laitière, où une partie des très jeunes (mâles vraisemblablement) est abattue. Cette sélection n'est cohérente que dans l'existence de sites consommateurs. À l'inverse, dans les grands sites de plein air, l'abattage montre une visée consommatrice double : viande et lait (très jeunes adultes au moment de l'optimum pondéral et d'une viande tendre, bêtes de réforme âgées). Les deux profils, tronqués séparément, sont complémentaires. Sans que l'on puisse déduire à ce stade la nature du rapport existant entre les deux types de sites (rapport marchand entre sites autonomes, déplacement ou partition du troupeau d'un village en des points spécialisés...), leur lien apparaît clairement. Pour les bovins, c'est à la fois semblable et différent. Les grands sites de plein-air montrent également une consommation carnée visant surtout les bêtes de moins de deux ans et même majoritairement de six mois à un an, alors que manquent les très jeunes et les adultes reproducteurs. Il est alors probable que les naissances ne se faisaient pas sur ces sites consommateurs. Or, si l'on dispose bien des grottes-bergeries pour cette étape chez les caprinés, aucun site actuellement étudié ne correspond à cela pour les bovins.

Sans entrer dans un long débat et pour en rester à notre propos dans ce cas du Chasséen rhodanien, on peut constater que l'archéologie parvient à approcher de façon très plausible la question de la complémentarité entre des sites et même, si on y ajoute les sites « de naissances » inconnus des bovins, au sein de tout un réseau complexe de relations fonctionnelles. Pour les problèmes de l'habitat qui nous guidaient, l'état des faits est très troublant. Les grands sites de terrasse, s'ils apparaissent centraux et majeurs pour les activités funéraires et cérémonielles, peut-être aussi dans un rôle de concentration et de redistribution alimentaire, ne se présentent pas du tout comme les villages de base du système de résidence et de vie domestique. On cherche vainement les maisons
Porche de la grotte-bergerie néolithique d'Antonnaire (Montmaur-en-Diois, Drôme). (Fig. 5) 


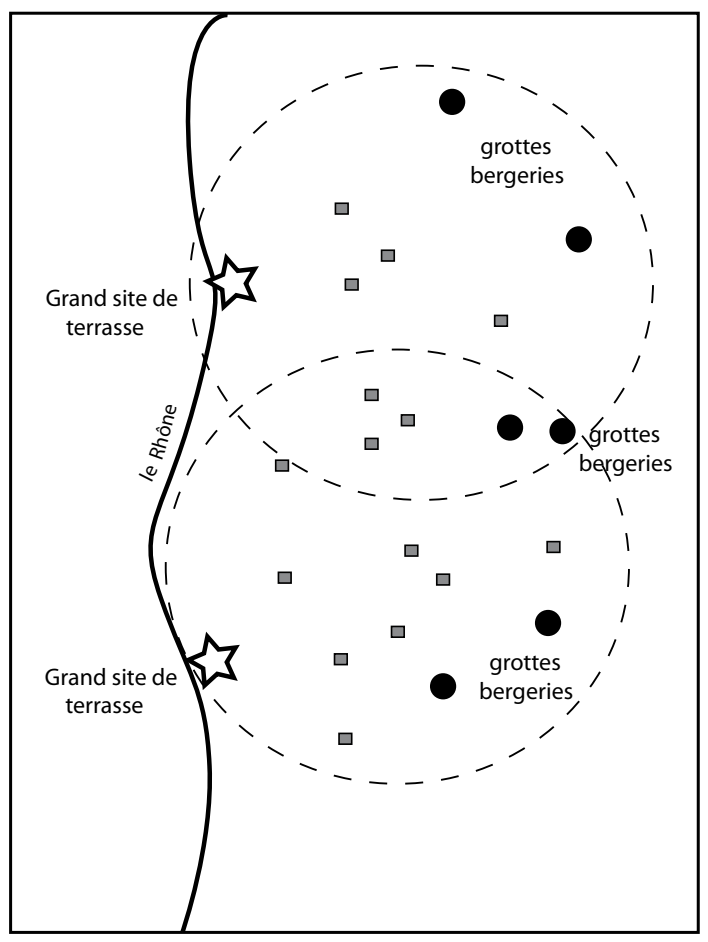

Plan de situation schématique des sites chasséens de la Drôme, entre Rhône (très grands sites) et moyenne montagne (grottes-bergeries)

Dans l'étage collinéen intermédiaire, de multiples sites de plein air, mal connus, peuvent correspondre aux villages manquant du réseau territorial (D.A.O. A. Beeching).

(Fig. 6) pérennes ou durables, voire même, dans l'hypothèse de leur non reconnaissance à la fouille, l'abondance des rejets qui devrait correspondre à la taille des sites. Avec leurs caractéristiques contraignantes et la faiblesse des traces d'organisation interne, les grottes et abris ne fournissent pas une alternative à cette carence. Ces gens étaient bien là, à ces deux endroits, mais devaient aussi être ailleurs. Deux hypothèses peuvent être formulées, sans preuves décisives pour le moment. Soit, il y avait des villages résidentiels de base à occupation longue (plusieurs années) selon le scénario le plus classique ; ces villages, actuellement inconnus, seraient dans la zone collinéenne mal documentée. Soit, pour des raisons climatiques et environnementales (Beeching \& al. 2000), l'économie de subsistance serait plutôt tournée vers le pastoralisme et la sédentarité serait moins grande, expliquant le grand nombre de sites reconnus de même que l'absence d'implantations lourdes dans cette zone géographique lors des fouilles anciennes et des trouvailles fortuites. Ce sont deux variantes de la même organisation territoriale, où grottes-bergeries et grands établissements de terrasses apparaissent d'une certaine manière comme des sites secondaires dans l'organisation résidentielle (Figure 6). Ceci expliquerait que tous deux n'offrent à l'analyse que des formes d'« habitats précaires » selon les principes de raisonnement définis au début.

\section{$\&$}

En conclusion, ces deux catégories d'exemples montrent la difficulté mais non l'impossibilité de raisonner sur le statut des habitats, à partir des données de l'archéologie. On y voit que, si la catégorisation des faits sur des bases matérielles quantitatives n'est pas absurde, en soulignant des différences notoires entre sites contemporains, elle peut par contre conduire à une bipolarisation hâtive et fausse entre gros et petits sites, sites majeurs et secondaires, producteurs et récepteurs, « donneurs d'ordres » et exécutants, etc., en occultant des données plus discrètes ou mal connues autant que des schémas explicatifs plus complexes. Opposer une forme d'implantation à une autre, fait courir le risque de les classer hiérarchiquement et de masquer la complexité du réseau de sites complémentaires. Une autre tentation doit être écartée : celle de la réduction des hypothèses explicatives aux scénarios les plus tranchés, également les plus à même de fournir les paradigmes séduisants que réclame les joutes académiques (tels que le front 
pionnier de néolithisation, l'opposition nomade-sédentaire, les explications univoques aux « collapses » de civilisation...). Il est souvent plus difficile d'accorder du poids aux vestiges ténus ou aux carences qu'aux évidences flagrantes. Au Proche-Orient, les implantations sédentaires en tells masquent peut-être une réalité plus diverse. Dans le bassin rhodano-alpin, on a peut-être mésestimé les implantations d'altitude et les sites « pauvres». Quand il peut être sollicité, l'habitat livre des informations importantes. Mais il ne peut tout montrer. Son absence ou sa pauvreté aussi sont révélatrices.

\section{NOTES}

Photo d'ouverture : Un paysage classique des Préalpes drômoises du Diois. Sous le plateau, au pied de la barre calcaire et surplombant la vallée de la Drôme, s'ouvre la grotte-bergerie néolithique d'Antonnaire.

1. Frédéric Abbès (UMR 5133 « Archéorient » du CNRS, Maison de l'Orient et de la Méditerranée, Lyon) mène depuis six ans, en co-direction avec Thaer Yartah (D.G.A.M. Alep, Syrie), des prospections et fouilles dans cette région aride. Les résultats en sont spectaculaires et novateurs. Je lui suis gré de pouvoir mentionner très brièvement quelques unes de ses données inédites et je le remercie vivement pour les illustrations qu'il m’a fournies.

2. Je remercie Danièle Stordeur pour la communication d'un cliché de son site de Jef el Ahmar.

3. Je remercie également Frédéric Cordier pour les informations concernant les fouilles de 2006 au Gournier. 


\section{RÉFÉRENCES}

Alarashi, H. 2005 Premières traces du nomadisme pastoral au Proche-Orient à la fin du Néolithique précéramique 7600-6400 avant J.-C.- État actuel de la recherche. Mémoire de Master. Université Lumière-Lyon 2.

Aurenche, O. \& Kozlowski, S.K. 1999 La Naissance du Néolithique au Proche-Orient ou le paradis perdu. Paris : Éditions Errance.

Bar Yosef, O. et Valla, F. (dir.) 1991 The Natufian Culture in the Levant. Ann Arbor, International Monographs in Prehistory. Archaeological Series 1 : Michigan.

Beeching, A. 1999 Quelles maisons pour les néolithiques méridionaux? Les cas rhodaniens examinés dans le contexte général. In A. Beeching et J. Vital (dir.) - Préhistoire de l'espace habité en France du Sud et Actualité de la Recherche, Actes des Premières Rencontres Méridionales de Préhistoire Récente, Valence, 1994. Travaux du Centre d'Archéologie Préhistorique, nº 1 : Valence, 29-61.

— 2003 Organisation spatiale et symbolique du rituel funéraire chasséen en moyenne vallée du Rhône : première approche. In E. Chambon \& J. Leclerc (dir.) Les pratiques funéraires néolithiques avant 3500 av. J.-C. en France et dans les régions limitrophes, Table-ronde SPF de Saint-Germain-en-Laye, juin 2001. Paris : Mémoire n XXXIII de la Société Préhistorique française, 231-239.

Beeching, A., Berger J.-F., Brochier J.-L. \& al. 2000 Chasséens : agriculteurs ou éleveurs, sédentaires ou nomades? Quels types de milieux, d'économies et de sociétés? In Sociétés et Espaces, Rencontres Méridionales de Préhistoire Récente, 3ème session, Toulouse, 1998. Toulouse : Ed. Archives d'Ecologie Préhistorique, 59-79.

Beeching, A. \& Brochier, J.L. 1990 Archéologie spatiale entre Rhône et Alpes du Sud. L'exemple du Néolithique chasséen, Bulletin du Centre Genevois d’Anthropologie, nº 2 .

Beeching, A., Binder, D., Blanchet, J.- C. \& al. (dir). 1991 Identité du Chasséen. Actes du colloque international de Nemours, 1989 Nemours : APRAIF, 1991 Mémoire du Musée de Préhistoire d'Ile-de-France nº 4.

Beeching, A., Brochier, J. L., Cordier, F., \& al. 2010 Le Gournier : historique des recherches et présentation d'un « grand site » chasséen en vallée du Rhône. In A. Beeching, E. Thirault \&J. Vital (dir.) : Économie et société à la fin de la Préhistoire \& Actualité de la Recherche, Actes des VIIe Rencontres Méridionales de Préhistoire Récente, Lyon - Bron, 2006, DARA n 34. Lyon : Maison de l'Orient et de la Méditerranée : 187-205.

Bréhard, S. 2007 Contribution archéozoologique à la connaissance de la fonction des grands sites de terrasse du Chasséen récent (début du IVe millénaire avant J.-C.) de la moyenne vallée du Rhône, dans leur contexte de Méditerranée nord-occidentale. Paris: Muséum national d'Histoire naturelle 2 vols (Thèse de doctorat).

Bréhard, S., Beeching, A. \& Vigne, J.-D. 2010 Sheperds, cowherds and site function on middle Neolithic sites of the Rhône valley : An archaeozoological approach to the organization of territories and societies. Journal of Anthropological Archaeology, 2 : 179-188.

Brochier, J.L., Beeching, A., Sidi Maamar, H. \& al. 1999 Les Grottes-bergeries des Préalpes et le pastoralisme alpin durant la fin de la Préhistoire. In A. Beeching (dir.) Circulations et identités cuturelles alpines à la fin de la Préhistoire. Programme CIRCALP 1997-1998 - Agence Rhône-Alpes pour les Sciences Humaines, Valence, 1999 (Travaux du Centre d'Archéologie Préhistorique de Valence, 2) : 77-114

Brochier, J.L. \& Beeching, A. 2008 Les Grottes-bergeries dans le système pastoral. In : Premiers bergers des Alpes. De la Préhistoire à l'Antiquité, Livret-catalogue de l'exposition. Grenoble : Musée Dauphinois, 69-73.

Cauvin, J. 1990 Nomadisme néolithique en zone aride : l'oasid d'El Kowm (Syrie). In P. Matthiae, M. Van Loon \& H. Weiss (dir.) Resurrecting the Past. Instanbul : Nederlends Historisch-Archaeologisch Instituut, 41-47.

Demoule, J.-P. (dir.) 2009 La Révolution néolithique dans le monde. Paris : Éditions du CNRS.

Francfort, H.-P. (dir.) 1990 Nomades et sédentaires en Asie Centrale. Apports de l'archéologie et de l'ethnologie. Paris : Éditions du CNRS.

Gourichon, L. 2004 Faune et saisonnalité : l'organisation temporelle des activités de subsistance dans l'Epipaléolithique et le Néolithique précéramique du Levant nord (Syrie). Lyon : Université Lumière-Lyon 2 (Thèse de doctorat). 
Helmer, D., Gourichon, L., Sidi Maamar, H. \& al. 2005 L'Élevage des caprinés néolithiques dans le SudEst de la France : saisonnalité des abattages, relations entre grottes-bergeries et sites de plein air. Anthropozoologica, nº (1) Muséum national d'Histoire naturelle de Paris : 167-189.

Molist Montana, M. 2001. Halula, village néolithique en Syrie du Nord. In J. Guilaine (dir.) Communautés villageoises du Proche-Orient à l’Atlantique (8000-2000 avant notre ère). Paris : Éditions Errance, 35-50.

Saintot, S., Le Barrier, C. \& al.2009 L'Habitat chasséen de Champ Madame et Artière-Ronzière à Beaumont (Puy-de-Dôme). Structuration, architecture et fonction du bâtiment 3. In A Beeching \& I. Senepart (dir.) De la maison au village. L'habitat néolithique dans le Sud de la France et le Nord-Ouest méditerranéen, Actes de la table-ronde de la Société Préhistorique Française (Marseille, Mai 2003). Paris : Mémoire XLVIII de la Société préhistorique française, 99-121.

Stordeur, D. 1999 Organisation de l'espace construit et organisation sociale dans le Néolithique de Jerf el Ahmar (Syrie, Xe-XIe millénaire av. J.-C.). In F. Braemer, S. Cleuziou \& A. Coudart (dir.) Habitat et Société, XIXe Rencontres Internationales d'Archéologie et d'Histoire d'Antibes, Antibes: APDCA, 131-149.

Stordeur, D. 2000 Jerf el Ahmar et l'émergence du Néolithique au Proche Orient. In J. Guilaine (dir.) Premiers paysans du monde. Naissances des agricultures. Paris : Éditions Errance, 33-60.

\section{RÉSUMÉ}

Habitats pérennes ou précaires au Néolithique. L'examen de deux séries d'exemples archéologiques, issus de contextes néolithiques du Proche Orient et de Moyenne vallée du Rhône, permet d'aborder la question de l'importance relative des sites et d'en montrer les pièges. La hiérarchisation de l'importance des traces offre une piste, mais fait courir le risque d'une polarisation interprétative dans le sens d'une complémentarité fonctionnelle simple. Seule une information plus complète sur les activités pratiquées donne, au-delà des évidences, les preuves des liens entre sites. Les notions de sites majeur et secondaires, d'habitats pérennes et précaires en sont relativisées, au profit de celles de réseau complémentaire et d'adaptation au milieu.

\section{ABSTRACT}

Perennial or precarious settlement in the Neolithic. Considering two neolithic archaeological examples coming, one from the Near East (Syria), the other from the Middle Rhône Valley (France), enables us to approach the question of the relativity of sites importance and points its defects. Treated on a hierarchical basis, the importance of traces and remains leads to conclude on first interprétations. This is dangerous, because of the risk to think only in the sens of a simple functional complementarity. Only, the complete information on the practised activities gives, beyond évidences, the proofs of the links between sites. Of this fact, notions of major and secondary sites, long lasting or precarious settlements are put in perspective in profit of notions of complementarity network and adaptations to the environment.

\section{MOTS CLÉS}

Archéologie, néolithique, habitat, pastoralisme, réseau de sites

\section{KEYWORDS}

Archaeology, Neolithic era, habitat, pastoralism, network of sites 